\title{
Minorías nacionales y medios de comunicación: una visión de Europa
}

\author{
Dra. Sara Núñez de Prado \\ Prof. Titular Historia del Periodismo y directora del Doctorado en Estudios Europeos de la \\ Universidad Europea de Madrid. Historiadora. Experta internacional del Consejo de Europa \\ para los temas de minorías nacionales. Miembro español del Comité Consultivo del \\ Convenio Marco para la Protección de las Minorías Nacionales (Consejo de Europa)
}

\section{RESUMEN}

Los medios de comunicación audiovisuales han abierto las ventanas de un mundo desconocido a amplias capas de la sociedad. Estos grupos sociales nunca habrían podido conocer lo que, por ejemplo, la televisión les ha mostrado. Pero esta realidad, positiva en sí misma, tiene una cara de sombra que no se puede olvidar. Éste es el problema que, tradicionalmente venimos definiendo como la capacidad de acceso a los medios de comunicación. Todo el mundo puede ver la televisión, pero no todas esas personas (o grupos sociales) tienen opción a salir por esa misma televisión. No suelen tener oportunidad de contar con unos minutos de emisión que les permita hacer al resto de la audiencia partícipe de sus cuitas. Y, además, precisamente por el hecho de que todo el mundo puede ver la televisión o escuchar la radio, se hace tan importante el poder tener unos minutos para poder ser visto y escuchado.

En este artículo no vamos a analizar las posibilidades reales, desde el punto de vista sociológico, del acceso de los distintos grupos sociales a los medios de comunicación, fundamentalmente los audiovisuales. Lo que queremos es hacer un breve estudio comparativo entre distintos estados de Europa de la cuota de presencia que en sus respectivos países tienen las minorías nacionales en los medios de comunicación. Una aclaración más. El término minoría nacional se utilizará en este trabajo siguiendo la definición que el Consejo de Europa ha ofrecido del mismo y la base documental del estudio, junto a otra serie de documentos diversos, serán los informes que los gobiernos de esos países han remitido al Consejo de Europa sobre el estado legal, tratamiento general en distintos ámbitos y presencia efectiva de sus minorías nacionales.

\section{ABSTRACT}

Audio-visual media have opened up the windows of an unknown world for a wide range of social layers. These social groups had never been able to know what, for instance, television has shown to them. But this reality, which is positive itself, has a shadow face that can not ever be forgotten. That is the problem which we have been traditionally defining as the capacity for media access. Everyone is able to watch television, but not all people (or social groups) are able to appear on television. They do not have the opportunity to enjoy some minutes of broadcasting which would them able to show the audience their own worries. In addition, and precisely because everybody is able to watch television or listening to radio, it is so important to appear on television or to be listened to on radio.

This paper is not an intend to analyse the real possibilities, from a sociological point of view, of the access of the different social groups to media, especially to the audio-visual media. What we will try to make is a brief comparative study of the diverse European states regarding the participation of national minorities on media. The concept "national minority" will be used 
in this paper following the definition of The European Council. Finally, the main material used for this study, along with other documentation, will be those government reports submitted to The European Council on the national minorities legal situation, general treatment as regards various spheres, and real participation on media.

Palabras claves: Minorías nacionales en Europa/Acceso a los medios de comunicación/Tolerancia/Consejo de Europa.

Key words: National minorities in Europe/Capacity for mass media access/Tolerance/European Council.

"La justicia es una construcción humana, y es dudoso que pueda ser realizada de una sola manera. En cualquier caso (...) yo pretendo añadir algo más que esto: que los principios de la justicia son en sí mismos plurales en su forma; que bienes sociales distintos deberían ser distribuidos por razones distintas, en arreglo a diferentes procedimientos y por distintos agentes; y que todas estas diferencias derivan de la comprensión de los bienes sociales mismos, lo cual es producto inevitable del particularismo histórico y cultural"

(Walzer, 1997:19)

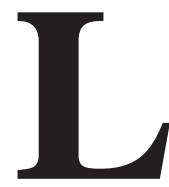

a sociedad actual se desarrolla en un esquema geográfico/cultural/político en el que la realidad es la casi inexistencia de países monoculturales. La práctica totalidad de los estados del mundo se enfrentan a una sociedad culturalmente diversa dentro de sus fronteras. En este sentido, es interesante distinguir entre aquellos países que pueden considerarse multinacionales de los que podrían llamarse multiétnicos (Kymlicka, W., 1996). En el primer caso se encuentran aquellos estados que han sido capaces de asimilar, ya por la fuerza, ya libremente, grupos nacionales en su seno que devienen minoritarios con el paso del tiempo. Estos grupos son los que entendemos como minorías nacionales, poseedores históricamente de una tierra propia, una lengua y unos valores culturales específicos. En cambio, cuando hablamos de pluralismo étnico, se hace referencia a la capacidad que un país tenga de asumir en su seno a importantes contingentes de inmigrantes. Estos grupos también mantendrán sus diferencias culturales o étnicas, pero carecerán del arraigo de la tierra propia dentro del estado que les acoge. La existencia, tanto de grupos multiétnicos como multinacionales en los estados nacionales, es lo que algunos autores han situado en el nivel vertical de las diferencias (POMIAN, 1990). Y en general parece que los procesos internos de los estados europeos en la actualidad tienden a minimizar dichas diferencias, caminando hacia la integración y la convivencia pacífica. Sartori profundiza en este tema haciendo referencia a lo que él llama 
cross-cutting cleavages (líneas de división cruzada). Y clarifica este concepto, diciendo que "de hecho la ausencia de cleavages cruzados es un criterio que permite por sí solo excluir del pluralismo a todas las sociedades cuya articulación se basa en tribu, raza, casta, religión y cualquier tipo de grupo tradicionalista (...) el pluralismo funciona cuando los cleavages, las líneas de división, se neutralizan y frenan por múltiples afiliaciones (y también lealtades), mientras que disfunciona, por así decirlo, cuando las líneas de fractura económicosociales coinciden, sumándose y reforzándose unas a otras (por ejemplo, en grupos cuya identidad es a la vez étnica, religiosa o lingüística). En este caso aún cabe asegurar la paz y la coexistencia social si hay elites consociativas (era el caso, por ejemplo, de Holanda). Pero la paz social está en peligro cuando las comunidades cerradas con cleavages coincidentes se convierten en invasoras y agresivas" (SARTORI, 2001:40).

La diversidad antes descrita, ya sea multinacional o multiétnica, y por encima de los cleavages descritos por el autor italiano, plantea una serie de cuestiones que los estados democráticos están obligados a resolver desde la tolerancia y la igualdad. Eso les lleva a la no siempre fácil tarea de armonizar los derechos de las minorías con los de las mayorías. En muchos casos, además, esta necesidad de equilibrio no siempre es sencilla de alcanzar. Los enfrentamientos entre los derechos de unos y otros, la conciliación de las necesidades, las aspiraciones de autonomía, etc. son muchas veces fuente de tensiones, incluso en sociedades en las que los principios democráticos están arraigados desde hace mucho tiempo.

\section{Las minorías nacionales y la necesidad de pluralismo ${ }^{1}$}

Históricamente, cuando hablamos de protección de minorías nos encontramos con dos concepciones básicas diferentes. Una de ellas, la más clásica, es de corte liberal y se apoya en la idea de que si existe una defensa efectiva de los derechos humanos para todos los individuos en general, no hace falta que se desarrolle una legislación específica de protección o promoción de derechos para grupos minoritarios. Esta concepción ha sido la predominante hasta fechas muy recientes. Así, por ejemplo, no encontramos ningún tipo de referencia específica a los derechos de los grupos minoritarios en textos legislativos tan importantes como la Declaración Universal de los Derechos Humanos de las Naciones Unidas o en la Carta de los Derechos Humanos del Consejo de Europa.

En los últimos años, frente a este concepto, se fue abriendo paso con fuerza otra idea que descansaba en el convencimiento de que la simple defensa generali-

1 En este trabajo nos vamos a centrar exclusivamente en las minorías nacionales, dejando a un lado la problemática de los inmigrantes que por sí sola sería objeto de otro estudio. 
zada de los derechos humanos no resolvía los problemas específicos de grupos minoritarios. Es más, se presentaba como necesaria una legislación específica encaminada a solucionar problemas singulares de estos colectivos. Por ejemplo, el uso de sus lenguas maternas, el derecho a ser educados también en sus tradiciones, etc. A partir del derrumbamiento de la antigua Unión Soviética todo este conglomerado de problemas salieron a la luz, muchas veces, incluso, con extrema virulencia. El nuevo rediseño del mapa de Europa puso de manifiesto que en la raíz de una gran parte de los enfrentamientos humanos habidos subyacían heridas no cerradas protagonizadas por grupos minoritarios nacionales. "Todo ello ha reforzado los procesos de devolución, regionalización o federalización, potenciando el renacer de viejas culturas locales que fueron poderosas y dominantes durante la Edad Media y el Ancien Régime. Incluso el proceso de construcción política de Europa parece dar nuevos aires a viejas regiones naturales, rotas por la rigidez de las fronteras estatales, y que reencuentran vínculos históricos. De este modo emerge un segundo multiculturalismo interno a la propia cultura occidental, por disgregación o diversificación" (LAMO DE ESPINOSA, 1995:62)

Es extraño encontrar hoy en Europa un país que no esté inmerso en algún tipo de proceso de multiculturalismo nacional. No sólo en la esfera de la antigua Unión Soviética, en donde algunos de los procesos han sido ejemplares, como el de los lituanos o los estonios. O tampoco sólo en los Balcanes, en donde desgraciadamente el multiculturalismo se ha cubierto de sangre, como en el caso de los serbios o los bosnios. Sino también en la sólida y tradicional Europa occidental, en donde la mayoría de sus países están viviendo rebrotes, más o menos importantes de multiculturalismo nacional. Estados como Gran Bretaña, Francia, España, Alemania o Italia, cuentan con minorías nacionales que reivindican sus derechos ya sea violenta (afortunadamente en los menos casos) o pacíficamente. Estos países se han visto abocados a enfrentarse a cuestiones de identidad que han sido debatidas intensamente en el seno de sus instituciones a lo largo de la década de los 90. En la otra cara de la moneda podríamos citar países como Islandia, quizás el más monocultural de toda la Europa occidental, junto a microestados como Liechenstein o San Marino.

Llegado este punto, ha ido tomando protagonismo una corriente que ampara la necesidad de que exista una legislación específica que defienda y promueva los derechos de las minorías nacionales. Encuadradas en ella, cabe citar iniciativas como la Carta sobre Derechos de las Minorías, aprobada por la Conferencia de Seguridad y Cooperación en Europa en 1991 o la Carta Europea para la Defensa de las Lenguas Minoritarias, adoptada en el seno del Consejo de Europa en 1992. Y, más recientemente y probablemente la que más arraigo e importancia ha alcanzado, el Convenio Marco para la Protección de las Minorías Nacionales, también del Consejo de Europa, aprobado en 1994 y que entró en vigor el 1 de 
febrero de 1998. Ha sido firmado por más de 40 países de los que la gran mayoría lo han ratificado ${ }^{2}$.

Uno de los grandes ausentes de este Convenio es Francia. El país galo no reconoce minorías nacionales en su territorio, motivo por el que no suscribió el Tratado. Y no deja de ser una postura curiosa cuando este país ha sido uno de los estados con más tradición inmigrante a lo largo de los últimos siglos. El siglo XIX francés conoció la llegada de grandes oleadas de extranjeros. Tanto es así, que entre 1890 y 1990 cerca de 3 millones de ciudadanos originalmente extranjeros accedieron a la nacionalidad francesa. Pero, a pesar de ello, Francia presenta un cuadro de gran homogeneidad cultural y linguística. Es decir, Francia se convierte en un claro ejemplo de "un país que puede incluir ciudadanos de muy diferentes orígenes sin que necesariamente se defina a sí mismo como una sociedad multicultural". Añade la misma autora, como aclaración a lo anterior, que "el término multiculturalismo tiene diferentes significados, dependiendo de la aproximación a la identidad nacional. Hay estados que pueden rechazar el multiculturalismo y a la vez alentar una muy rica producción cultural, mientras que otros estados que profesan el multiculturalismo, transforman las culturas minoritarias en folklore fosilizado" (COSTA-LASCOUX, 1995:158).

El Convenio Marco, que consta de 32 artículos, pone de manifiesto en su propio texto que la protección de las minorías nacionales, así como de sus derechos y libertades, es parte integrante de la protección internacional de los derechos humanos. En su parte introductoria recuerda que la agitada historia de Europa nos ha puesto de manifiesto que la protección de las minorías nacionales es esencial para la estabilidad, la seguridad democrática y la paz. Y añade la necesidad de que las sociedades democráticas no se conformen sólo con mostrar respeto por las diferencias étnicas, culturales, religiosas o lingüísticas, sino que deben propiciar las condiciones imprescindibles para permitir que dichas diferencias puedan ser expresadas, preservadas y desarrolladas desde la propia identidad. Advierte, asimismo, que crear ese clima de tolerancia y diálogo entre los diversos grupos para permitir la diversidad cultural, no puede confundirse con división, si no que, muy al contrario, hay que tener presente que lo que hace es enriquecer a las sociedades. Y, finalmente, pone de manifiesto que alcanzar esos grados de tolerancia y respeto, a la vez que de promoción, no es una labor que pueda hacerse exclusivamente entre estados. Es un trabajo que requiere, además de la transfron-

2 Los estados firmantes han sido los siguientes: Albania, Alemania, Armenia, Austria, Azerbaijan, Bélgica (no ha ratificado), Bosnia Herzegovina, Bulgaria, Croacia, Chipre, Rep. Checa, Dinamarca, República de Eslovaquia, Eslovenia, España, Estonia, Finlandia, Georgia (no ha ratificado), Grecia (no ha ratificado), Hungría, Islandia (no ha ratificado), Irlanda, Italia, Letonia, (no ha ratificado), Liechtenstein, Lituania, Luxemburgo (no ha ratificado), Republica de Macedonia, Malta, Moldavia, Países Bajos (no ha ratificado), Noruega, Polonia, Portugal, Reino Unido, Rumania, Federación de Rusia, San Marino, Suecia, Suiza, Ucrania y República Federal de Yugoeslavia. 
terización, una labor interna que se desarrolle en el ámbito tanto nacional, como regional y local.

El Convenio Marco cuenta con un mecanismo de comprobación del grado de implementación que el acuerdo internacional tiene en los países signatarios del mismo. Ese mecanismo es abierto, en el sentido de que sólo establece una serie de normas generales que deben ser desarrolladas por el Comité de Ministros del Consejo de Europa. Los estados firmantes tienen la obligación de enviar un informe al Secretario General en el que se especifique el grado de implementación del Convenio Marco en el país y las medidas que se han tomado o piensan tomarse para llegar a conseguir los principios establecidos por el Convenio. El estudio y evaluación de dicho informe está a cargo del Comité Consultivo del Convenio Marco para la protección de las minorías nacionales. Este Comité está formado en la actualidad por 18 miembros independientes (el número no puede ser inferior a 12, ni superior a 18). Deben ser reconocidos expertos en el campo de la defensa y protección de las minorías nacionales. Son propuestos por los estados firmantes y elegidos por el Comité de Ministros. El Comité Consultivo evalúa los informes presentados y emite un informe con las recomendaciones que considera oportunas para que el estado en cuestión mejore la situación de sus minorías nacionales. Este informe es presentado ante el Comité de Ministros. Si éste lo aprueba, dará traslado de dichas recomendaciones al país para que tome las medidas oportunas. Además, el país deberá informar posteriormente de los progresos alcanzados.

\section{Medios de comunicación e identidad}

Muy conectado con el contexto anteriormente descrito, los medios de comunicación son una de las herramientas más importantes con las que cuenta el estado y la sociedad para enfrentarse a este tipo de realidades. Los mass media tienen un papel teñido de responsabilidad en la formación de actitudes de cara a la mayoría de la población que les obliga a comportarse como adalides de la tolerancia, la igualdad y la convivencia en paz. Además, uno de los elementos básicos, imprescindibles, de las sociedades democráticas, para que puedan ser consideradas así, es la existencia de pluralismo en los medios de comunicación.

A lo largo de la historia de la comunicación los hombres, individualmente, o las organizaciones de todo tipo, en su conjunto, han luchado por conseguir, primero, y por consolidar después, la libertad de expresión y de prensa y con ello, asentar los primeros escalones del pluralismo. Ahora bien, el reconocimiento de la libertad de expresión y de prensa y la garantía legal de la misma, incluso el ejercicio efectivo de ella, no aseguran por sí solas la existencia del pluralismo. Para que éste exista deben darse una serie de cuestiones añadidas. Primero, hay que garantizar el acceso de los diferentes grupos sociales a los medios de comunicación. Segundo, hay que regular y contrarrestar desde los poderes públicos la posible excesiva concentración de los medios de comunicación. Y tercero, hay que 
garantizar la existencia de ese pluralismo a diferentes niveles: nacional, regional y local. Es decir, hablamos de regular - que no limitar- el poder de los medios de comunicación desde las leyes y garantizar la presencia de todos los grupos sociales en ellos. Materia ésta que se convierte en la columna vertebral para garantizar la diversidad mediática, asegurar la libertad y promover la libre competencia.

Por lo que se refiere al tema de la concentración y teniendo presentes las limitaciones legales y los imperativos económicos, tradicionalmente se distinguen tres modelos de concentración: el horizontal, el vertical y el diagonal. No se puede olvidar que en estos temas, las leyes que regulan los medios de comunicación suelen ir íntimamente relacionadas con las que reglamentan la competencia en el ámbito de los mercados. Y además, ambos tipos de regulación suelen ser aplicables de una forma paralela, aunque esto varía en función de los países y depende también de que las leyes sobre comunicación sean consideradas generales, caso francés o italiano, por ejemplo, o especiales, como en Gran Bretaña.

Si subimos un escalón y nos movemos en el ámbito de la Unión Europea, cuando se habla de concentración de medios se aplican las leyes sobre competencia, que tienen prioridad sobre las leyes nacionales. Es más, los estados nacionales, por lo que respecta al amplio mercado europeo, solo mantienen responsabilidad plena en aquellos ámbitos en los que la Unión Europea permanece inactiva o en aquéllos en los que el propio interés nacional sea prioritario. En este segundo caso, la decisión tomada siempre tendrá que mantenerse dentro de los límites marcados por la Unión Europea, que ejercerá lo que podríamos denominar un control marginal.

Independientemente de todo lo anterior, y dentro de estas configuraciones generales permitidas por las leyes, se perfila la cuestión de dónde colocar la línea que separa los procesos de concentración permisibles, de aquéllos que se colocan más allá de lo social y políticamente conveniente.

\section{Minorías nacionales y presencia comunicacional: algunos ejemplos por pai- $\operatorname{ses}^{3}$}

En relación con el primer punto al que nos referíamos en el apartado anterior, el acceso de todos los grupos sociales a los medios de comunicación, éste se convierte en el asunto central de nuestro trabajo. Las constituciones de los países democráticos suelen garantizar el acceso teórico de toda la sociedad a los

3 La elección de los países se ha realizado pensando en ofrecer un abanico de ejemplos que pusiese de manifiesto distintas concepciones (dentro del modelo general) a la hora de enfrentar los países las cuestiones de las minorías nacionales. Obviamente, todos los que están no cubren las diferentes posibilidades, pero dentro del espacio disponible, creemos que el objetivo se ha cumplido. Y en cuanto a los datos que se recogen en este estudio, siempre que no se especifique otra cosa, están extraídos de los diferentes informes que los distintos países han remitido al Comité de Ministros del Consejo de Europa. 
medios. Y lo mismo ocurre con las leyes específicas sobre comunicación que existen en determinados países del mundo políticamente democrático. Y si acotamos más aún y nos fijamos específicamente en el tema de las minorías nacionales, suelen existir afirmaciones generalizadas, encaminadas a garantizar la igualdad de acceso $^{4}$ a los medios entre todos los ciudadanos, sean mayoría o minorías ${ }^{5}$.

\section{ITALIA (Referencia: ACFC/SR (99) 7)}

Este país mediterráneo sólo reconoce minorías lingüísticas en su territorio, lo que supone que un grupo social minoritario como el de los gitanos queda fuera de cualquier tipo de presencia efectiva social, comunicacional o de otro orden ${ }^{6}$. La propia Constitución italiana, en su artículo 6, hace referencia a la protección de las minorías lingüísticas, las cuales serán objeto de normas específicas. Además, hay que añadir el artículo 2, que garantiza los derechos fundamentales y el artículo 3 que se refiere a la igualdad entre todos los ciudadanos italianos, sin ningún tipo de discriminación.

Las comunidades lingüísticas reconocidas son las siguientes: alemana, albanesa, griega, eslovena y croata, franco-provenzal, francesa, occitana, catalana, ladina, sarda y friulana ${ }^{7}$. Pero hay que tener en cuenta que no todas las lenguas minoritarias reconocidas reciben el mismo trato. Por ejemplo, puede hablarse de cooficialidad de lenguas en el Norte del país. Así, en el valle de Aosta (noroeste de Italia) conviven el italiano y el francés y en el Trentin-Haut-Adige, lo que es el Tirol italiano (centronorte de Italia), el alemán y el italiano. En esta zona se ubica también la minoría ladina, pero su situación es diferente a la alemana. Asimismo, tiene un tratamiento especial, sin llegar a la cooficialidad, el esloveno,

4 Cuando hablamos de igualdad de acceso real nos estamos refiriendo a unas magnitudes establecidas en función de la presencia, el número, la dispersión/concentración geográfica, etc...

5 En este tema, aún moviéndonos en el ámbito de los países democráticos, hay que hacer diversas distinciones. Así, encontramos países que tienen y reconocen minorías nacionales en sus respectivos territorios (realmente la gran mayoría). Dentro de éstos, no obstante, hay que tener en cuenta que no todos ellos identifican el mismo tipo de minorías, o bien admiten como minorías nacionales a unos grupos sí y a otros no. Además, tampoco se puede olvidar que no todos los países han firmado y ratificado el Convenio Marco sobre la Protección de las Minorías Nacionales (Consejo de Europa). Ello supone que no todos los grupos reconocidos como minorías nacionales tienen universalmente acceso a los mismos derechos. También encontramos países, como España, que aunque no acepta la existencia de grupos de minorías nacionales en su territorio, debido a que todos los españoles tienen exactamente los mismos derechos ante la ley según la Constitución, sí acepta la existencia de un grupo diferenciado en suelo español, el de los gitanos. Y, en último lugar, existen países que consideran que en sus estados no existen minorías nacionales.

6 El caso de los gitanos en Italia es, además, bastante peculiar, ya que debido al nomadismo que caracteriza en la mayoría de los casos a este grupo social, tampoco tienen acceso a la nacionalidad italiana, aún habiendo nacido en el territorio del país. Por ello, la situación general de los gitanos italianos suele ser muy precaria en todos los niveles.

7 Algunos informes incluyen a los sicilianos como minoría, pero el realizado por el propio gobierno italiano no los nombra. 
en la zona del Froul-Vénétie Julienne (nordeste de Italia). En todas estas zonas, existe un estatuto especial de autonomía que es el que garantiza esa situación de privilegio especial. Pero fuera de estos casos, el resto de las lenguas minoritarias reconocidas no están especialmente protegidas. Es interesante señalar que en este terreno, como en otros muchos, se ponen claramente de manifiesto las diferencias existentes entre el Norte y el Sur del país.

Numéricamente la minoría más importante es la sarda, con más de 1.500 .000 personas reconocidas, lo que supone en torno al 2,7\% de la población total. Le siguen los friulanos, con el 1\%. A partir de esta cifra el resto de las minorías, no llega al 1\%, siendo la más numerosa de éstas la alemana/tirolesa, con un 0,5\%. Aunque la Constitución italiana no reconoce a los gitanos como minoría, éstos están en torno al 0,15 o ,0,17\% de la población, en una medida muy parecida a los eslovenos o albaneses (World Directory of Minorities, 1997). Precisamente, con referencia a los gitanos, el Informe Anual sobre el Desarrollo de los Derechos Humanos, elaborado por la International Helsinki Federation for Human Rights (IHF) en el año 1999, decía que este colectivo "ha sido víctima de la violencia policial y de una penetrante discriminación racial en virtualmente todas las esferas de la vida pública". Y continúa más adelante, "de acuerdo con el informe del Centro Europeo de los Derechos de los Gitanos (ERRC), los gitanos no disfrutan de los mismos derechos que la mayoría de la población. Están habitualmente sujetos, irremediablemente, a la violencia policial, motivada por cuestiones raciales y están discriminados con respecto a un amplio abanico de derechos, incluidos los derechos a un igual tratamiento ante la ley por parte de las autoridades y los tribunales, el derecho a la libertad de residencia, alojamiento, educación y acceso a los servicios públicos" (IHF, 1999:132-133).

El acceso de los grupos minoritarios a los medios de comunicación se planifica también exclusivamente desde esa perspectiva lingüística, sin tener en cuenta otro tipo de consideraciones. Sobre el papel, gracias a un acuerdo con la RAI, las minorías linguísticas tienen garantizado el acceso a los medios de comunicación. Pero la realidad es que más allá de los alemanes, franceses y eslovenos, que sí cuentan con una programación específica, el resto tiene una presencia más testimonial y teórica que efectiva. Así, en el valle de Aosta, en Bolzano y en Trieste, los estudios de la RAI elaboran directamente en sus lenguas respectivas informativos diarios y programas de contenido más general, también con una periodicidad diaria, referidos, principalmente, a la vida cultural. Todos ellos emitidos por radio y por televisión. En el resto de los casos, aunque existen proyectos a corto plazo, de momento en los medios radiotelevisivos sólo existe una programación difusa y sin periodicidad predeterminada.

En conjunto, puede concluirse con la idea de que los medios de comunicación en Italia son prácticamente italoparlantes. La programación en otras lenguas (alemán, francés y esloveno, sobre todo) constituyen más una excepción que otra cosa. 
Finalmente, hay que tener en cuenta que hasta la llegada del gobierno de S. Berlusconi, Italia estaba viviendo un proceso de cambio legislativo en la protección y promoción de sus minorías nacionales. Existían varios proyectos de ley muy avanzados, algunos tan importantes como el Proyecto 3366 para la Protección de las Minorías Lingüísticas. Pero realizar una evaluación de este proceso a estas alturas todavía es prematuro.

\section{HUNGRIA (Referencia: ACFC/SR (99) 10)}

En una posición opuesta al caso anterior, tenemos a Hungría, país que reconoce hasta trece minorías nacionales o étnicas distintas, de las que la más importante es la de los gitanos, con una población estimada de en torno a 600.000 personas ${ }^{8}$. Los derechos de todos estos grupos están reconocidos constitucionalmente (Art. 68), así como su participación en la vida pública, el establecimiento de órganos propios de gobierno nacionales y locales, la defensa y fomento de su propia cultura, el uso de sus lenguas y la educación en las mismas.

Por lo que a los medios de comunicación se refiere, la Broad Casting Act insiste en la necesidad de realizar programas que representen la vida y la cultura de los grupos minoritarios, considerando estos programas como de servicio público. También, según la misma ley, todos los programas que son clasificados como de servicio público están obligados a emitir información en las diversas lenguas minoritarias. A su vez, la Minority Act estipula que el servicio público de radio y televisión tiene la obligación de regular, preparar y transmitir programas específicos para las minorías nacionales o étnicas. Estos programas, además, tendrán que prestar especial atención en difundir los valores culturales de dichas minorías. Esta obligación se llevará a cabo tanto a nivel nacional, como regional o local. La elección del espacio de emisión depende de la presencia geográfica de los grupos minoritarios. Asimismo, estas minorías tendrán un representante en los organismos públicos mediáticos, elegido a través de los consejos de autogobierno de las propias minorías 9 .

La Hungarian TV Public Foundation Advisory Board, además, anima a las diversas minorías a emitir programaciones en sus propias lenguas y referidas a sus distintas realidades, utilizando la televisión por cable. Precisamente para subvencionar en parte este tipo de programación se destinaron en el año 199815 millones del presupuesto total.

8 Estos grupos reconocidos son (en orden de importancia): gitanos, alemanes, eslovacos, croatas, rumanos, polacos, serbios, eslovenos, búlgaros, griegos, armenios, ucranianos y rutenos. En total, se estima que Hungría cuenta con una población minoritaría que se cifra entre los 800.000 y 1.100 .000 personas sobre poco más de 10 millones de habitantes.

9 Estos organismos son los siguientes: Hungarian Radio, Hungarian TV y Hungarian TV Public Foundation Advisory Board. 
La programación televisiva dedicada a las minorías nacionales dio comienzo en Hungría en 1978. Por aquellas fechas, se realizó una programación específica para los croatas, los alemanes, los serbios y los eslovacos. Un nuevo paso se dio en 1982, cuando comenzaron a emitirse algunos de estos programas en las propias lenguas minoritarias. Y desde 1998, la televisión húngara emite programación para los trece grupos minoritarios reconocidos en la Minority Act, según la distribución que puede verse en el cuadro adjunto. La programación es preparada desde cuatro ciudades diferentes: Budapest, Pecs, Szeged y Szombathely, dependiendo de la presencia geográfica de las minorías.

Por lo que se refiere a la radio, existe programación en las lenguas minoritarias nativas desde 1998. Con un total, entre todos los programas, de en torno a 100 horas/día. La distribución puede verse también en el mismo cuadro:

\begin{tabular}{|l|l|l|}
\hline GRUPO MINORITARIO & PROGRAMACIÓN TV & PROGRAMACIÓN RADIO \\
\hline Gitanos & 25 minutos/semana & 3 horas/semana \\
\hline Croatas & 25 minutos/semana & 14 horas/semana \\
\hline Alemanes & 25 minutos/semana & 14 horas/semana \\
\hline Rumanos & 25 minutos/semana & 14 horas/semana \\
\hline Eslovacos & 25 minutos/semana & 14 horas y media/semana \\
\hline Serbios & 25 minutos/2 veces a la semana & 10 horas y media/semana \\
\hline Eslovenos & 25 minutos/2 veces a la semana & 1 hora/semana \\
\hline Búlgaros & 25 minutos/mes & 30 minutos/semana \\
\hline Griegos & 25 minutos/mes & 30 minutos/semana \\
\hline Armenios & 25 minutos/mes & 30 minutos/semana \\
\hline Polacos & 25 minutos/mes & 30 minutos/semana \\
\hline Rutenos & 25 minutos/mes & 30 minutos/semana \\
\hline Ucranianos & & 30 minutos/semana \\
\hline
\end{tabular}

A todo lo anterior hay que añadir una serie de programas soportados por los gobiernos locales que se transmiten sólo en el ámbito regional o local por diversas televisiones. Y además, hay que tener en cuenta que la programación que se emite por onda terrestre en el canal MTV 1, en horario de tarde, es repetida vía satélite los sábados por la mañana.

Según los datos que obran en nuestro poder, puede decirse que en el caso de Hungría los derechos a tener presencia comunicacional en la sociedad por parte de los grupos minoritarios están asegurados y protegidos. Es más, el "country report" que en 1997 realizó la Comisión de la Unión Europea, concerniente a la aplicación en el país de los principios comunitarios, así lo constató. Ahora bien, la realidad choca con esta visión teórica, entre otras cosas, debido al propio progreso, muy difícil, del país. La situación general no siempre ha permitido 
desarrollar en su totalidad los principios básicos recogidos legalmente. También hay que señalar que existen problemas de integración importantes en la sociedad húngara, sobre todo en relación con la población gitana ${ }^{10}$.

\section{AUSTRIA (Referencia: ACFC/SR (2000) 3)}

Austria es un país que desde las últimas elecciones está en el punto de mira de los países democráticos, de ahí el interés que ha pasado a tener en estos temas. El 3 de octubre de 2000 el FPÖ (Partido Liberal de Austria) consiguió unos resultados lo suficientemente buenos como para situarlo en posición de firmar, en febrero del 2000, un acuerdo con el partido de centro/derecha ÖVP (Partido Popular de Austria). Dicho acuerdo le permitía acceder a un gobierno de coalición en el que colocaba como vice-canciller a uno de los suyos (Susanne Riess-Passer). Las relaciones con la Unión Europea, a partir de ese momento, devinieron muy difíciles. Tanto es así, que a finales de julio del 2000, los catorce estados decidieron enviar una comisión de expertos ${ }^{11}$ para estudiar in situ el pasado del FPÖ, con especial atención en materia de derechos humanos.

Las conclusiones del informe, presentadas el 8 de septiembre del 2000, no fueron desfavorables, por lo que la Unión Europea decidió levantar, días después, las sanciones que se le habían impuesto a este país. El informe terminaba diciendo que, si bien, el FPÖ había explotado e impuesto sentimientos xenófobos durante la campaña electoral que habían creado un clima en el que determinadas expresiones contrarias a ciertas minorías y a emigrantes en general aparecían como habituales, la situación tenía visos de haberse serenado. Añadía en el apartado III, referido a las Conclusiones Generales, lo siguiente: "el gobierno austriaco está comprometido con los valores europeos. El respeto del gobierno austriaco, en particular por los derechos de las minorías, refugiados y emigrantes, no es inferior al de otros estados miembros de la Unión Europea. La situación legal de las tres áreas mencionadas alcanza los estándares aplicados en otros estados miembros de la Unión Europea. En algunas áreas, particularmente en las concernientes a los derechos de las minorías, el estándar austriaco puede ser considerado más alto que los aplicados en algunos otros países de la Unión Europea". Agregaba que "el FPÖ tenía también que intentar suprimir críticas a través de un uso continuado de procedimientos liberales" (puntos 110, 108 y 112 respectivamente).

10 El gobierno húngaro es consciente de ello y como se constata en los diversos informes, memorandos... enviados al Consejo de Europa, está poniendo en marcha una serie de acciones a medio y largo plazo para paliar esta situación. Así, se está preparando - se encuentra en período de discusión- un plan de estrategia que, con probabilidad, será dado a conocer y aprobado en el siguiente curso parlamentario.

11 Formaron esta comisión Martii Ahtisaari (ex-presidente de Finlandia), Jochen Frowein (Instituto MaxPlanck y antiguo Vicepresidente de la Comisión Europea sobre Derechos Humanos) y Marcelino Oreja (antiguo Secretario General del Consejo de Europa). 
El Acuerdo Marco para la protección de las Minorías Nacionales entró en vigor en Austria en julio de 1998. La ley sobre minorías (Volksgruppengesetz) define a los grupos minoritarios como "nacionales austriacos viviendo y residiendo en partes del territorio federal, cuya lengua madre no es el alemán y que tienen sus propias tradiciones y folklore" (Fed. Law Gazette No 396/1976, Sección 1, parágrafo 2). Pero, lo que esta ley no hace es decir qué grupos son los considerados como minorías nacionales. Para ello hay que recurrir a los órganos de gobierno que se dedican a estos temas. Así, a partir del Consejo Federal de Minorías Nacionales (Volksgruppenbeiräte) (Fed. Law Gazette No 38/1977), se puede decir que el gobierno austriaco reconoce en su territorio a los siguientes grupos minoritarios: croatas (en la región de Burgenland), eslovenos, húngaros, eslovacos y gitanos, aunque ninguno de estos grupos alcanza una presencia importante (no llegan al 1\% de la población cada uno de ellos).

Por lo que se refiere al acceso de dichos grupos minoritarios a los medios de comunicación, existe una ley de 1985, la llamada Ley de Promoción de la Prensa (Presseförderungsgesetz) que facilita éste así como la promoción de los grupos minoritarios en los medios de comunicación. Hay que añadir la Broadcasting Act, que obliga a la Austrian Broadcasting Corporation (ORF) a emitir un programa que favorezca y promocione la coexistencia democrática entre todos los grupos sociales (Sección 2, parágrafo1, subparágrafo 2).

Para cumplir con los mandatos legales anteriores, se creó el programa "Heimat, fremde Heimat" (Hogar, extraño Hogar) que está centrado en los grupos minoritarios nacionales y en los emigrantes. Se inició en 1989, con frecuencia semanal y una hora de duración. Se emite los domingos en horario de medio día y se repite, resumido, el sábado siguiente a primeras horas de la mañana. En el primer caso por el canal 2 y en el segundo, por el tercer canal. Parte se realiza en alemán y el resto en lenguas minoritarias, pero con subtítulos en el idioma germano. A la misma hora, los mismos días y con la misma frecuencia, "Heimat, fremde Heimat" es sustituido en Burgeland y en Carinthia por otros programas, de las mismas características, pero específicos para la minoría croata y la minoría eslovenia, respectivamente. En el caso de los croatas, se llama "Dobar dan Hrvat" (Hola croatas) y en el de los eslovenos, "Dober dan Koroska" (Hola Carinthia). Éste último se emite, además, por el canal 1 de la RTV Slovenija. Y desde 1990 existe un programa especial para la minoría húngara, Adj isten magyarok" (Hola húngaros), pero que sólo se emite cuatro veces al año y con una duración de 30 minutos cada vez. Como puede observarse sólo leyendo los títulos, todos ellos están realizados en las lenguas propias de cada minoría. A lo anterior hay que añadir una serie de programas que se emiten a través de la televisión por cable y, además, la posibilidad que existe en zonas fronterizas de captar vía satélite la programación de otros países (aunque en este caso no se puede considerar programación específica de grupos minoritarios, obviamente). 
En la producción radiofónica los tiempos empleados en programas especiales para los grupos minoritarios es bastante más extenso que en la televisión. Por ejemplo, en la zona de Burgeland el 9\% de la programación está dedicada a las minorías croata y húngara, lo que da una media de unos 40 minutos diarios para los croatas y de unos 20 minutos diarios para los húngaros. En la zona de Carinthia, con los eslovenos, la situación es similar. Se alcanza en torno al $10 \%$ de la programación de carácter específico, lo que se traduce en unos 55 minutos diarios. Y desde 1992 existe también programación, aunque en menor cuantía en otras áreas. Así, la Neues Radio Wien emite 30 minutos todos los domingos por la mañana, dedicados a los grupos minoritarios, en los que se incluye folklore, entrevistas, noticias locales... Una particularidad de este programa es que está hecho en Alemania. Y desde el año pasado, la Radio Romano Centro, patrocinada por la Romano Centro Association, emite un programa semanal, también de 30 minutos, en alemán y romaní, pensado para la minoría gitana. Aunque parece ser que esta emisión tiene muy poca audiencia. Todos estos programas radiofónicos, además, pueden seguirse a través de Internet. A todo lo anterior, hay que añadir una serie de programas de radio que se emiten vía radios privadas. Entre éstas destaca la Agora Korotan Lokalradio Gmbh que emite con licencia desde 1998 para la zona de Carinthia y que el 50\% de su programación la realiza en esloveno. Y también la llamada Radio MORA (Multilingual Open Radio) que trabaja en la zona de Burgeland y que, además de en croata, emite en húngaro y en romaní. Todas estas radios tienen subvenciones públicas en diferente cuantía.

En general, puede concluirse que el acceso de los grupos minoritarios nacionales en Austria está suficientemente garantizado, aunque no existe una igualdad de condiciones entre todos ellos. No obstante, también hay que tener en cuenta en el caso concreto de este país, la imagen que desde los medios de comunicación, en su programación general, se da de los grupos minoritarios. El tratamiento que estos grupos reciben no siempre es correcto $\mathrm{y}$, a la vez, tampoco parece que exista un compromiso claro por parte de los medios en promover a todos los grupos minoritarios por igual. Las conclusiones del segundo informe sobre Austria (junio de 2000) realizado por la Comisión Europea contra el racismo y la Intolerancia (Referencia: CRI (2001) 3) en lo que al tratamiento de estos grupos en los medios de comunicación se refiere es claro (el comentario se centra en los periódicos de tirada nacional, pero es extrapolable a la radio y la televisión): los principales medios regularmente informan de una manera que contribuye a crear una atmósfera de hostilidad y rechazo hacia los miembros de los grupos minoritarios (...) a menudo retratan a estos grupos utilizando estereotipos negativos y explotando sensacionalísticamente incidentes en los que se ven envueltos miembros de grupos minoritarios" (pág. 15, punto 33$)^{12}$.

12 Hay que tener en cuenta que estos informes se fijan principalmente en los grupos de emigrantes y en lo que en los últimos tiempos han comenzado a denominarse "nuevas minorías". Es decir, grupos en origen de emigrantes, pero que por arraigo pueden convertirse en minorías nacionales pasado el tiempo. 


\section{ALEMANIA (Referencia: ACFC/SR (99) 17)}

Alemania es un país que puede considerarse un modelo de concentración horizontal y dentro de éste específicamente encuadrado en la alternativa conocida como "The Audience Share Model". Pero no es un modelo puro, ya que dentro de la horizontalidad se presentan elementos típicos de los otros dos (verticalidad y diagonalidad). Pero, en última instancia, esos elementos extraños siempre se utilizan para dar una respuesta desde la horizontalidad.

La constitución alemana reconoce a todos los grupos sociales alemanes, absolutamente y sin restricciones, todos los derechos y libertades recogidos en esta ley básica. A ella hay que añadir las leyes específicas de cada länder en los que existen establecidas minorías nacionales. Además, en el caso concreto de Alemania, el Convenio Marco de Defensa de las Minorías Nacionales fue aprobado por la Federal Act de 22 de julio de 1997. Esta ratificación supone que el acuerdo internacional se considera y tiene valor de ley federal. En este repaso legal, hay que añadir el artículo 5 de la Constitución, que reconoce el derecho a la libertad de expresión y el artículo 10 de la European Convention on Human Right, también con rango de ley federal ${ }^{13}$.

Las minorías nacionales reconocidas en territorio alemán, son la danesa, tradicionalmente asentada en Schleswig desde hace más un siglo y la serbia, también con una vieja tradición histórica que la asienta en Saxon y Brandemburgo (en ambas zonas hay pueblos de clara mayoría serbia). Un tercer grupo importante es el formado por la minoría frisona, repartidos entre el área de East Frieslan y el norte de Odemburg. Y, finalmente, está reconocido un grupo minoritario formado por los pueblos sinti y gitano, en territorio alemán desde los siglos XIV y XV, pero cuya presencia, al contrario que en los casos anteriores, se encuentra muy dispersa. A pesar de esa diseminación, puede afirmarse que la presencia de estos grupos es más importante en las grandes ciudades que en los pueblos y dentro de aquéllas destacan capitales como Hamburgo, Berlín, Düsseldorf y Colonia. Es decir, las grandes ciudades de los llamados "old länder".

Centrándonos en los medios de comunicación. En este país hay que estar a lo establecido por cada länder, ya que el estado federal tiene prohibido, expresamente por la Constitución, intervenir de cualquier modo en los contenidos de los programas internos ${ }^{14}$. Evidentemente, las diferentes Land Broadcasting Act y los State Treaties tienen taxativamente prohibido cualquier tipo de contenido xenófobo, racista, etc., a la vez que deben, no sólo respetar, sino también, promover los

\footnotetext{
13 El sistema alemán de inserción de las normas internacionales ha seguido siempre una concepción dualista tradicional, que sigue pesando mucho aún hoy, aunque las leyes alemanas se muestran en estos temas extremadamente progresistas. Debido a ello, toda norma internacional ratificada por el parlamento alemán es asumida en el ordenamiento germánico con el rango de ley federal.

14 En cambio, la programación para el exterior es competencia exclusiva del estado federal.
} 
intereses de los diferentes grupos minoritarios. El control sobre todo ello se realiza a través de una serie de instituciones supervisoras, como el Sound Broadcasting Board o el TV Board, formados por representantes de diversos grupos sociales y políticos. Y, también muy importante en este tipo de tareas es el Central Council of German Sinti and Roma ${ }^{15}$.

Centrándonos en la presencia efectiva de los grupos minoritarios reconocidos constitucionalmente en la programación de radio y televisión, ésta puede verse en el cuadro adjunto:

\begin{tabular}{|c|c|c|c|}
\hline $\begin{array}{l}\text { GRUPO } \\
\text { MINORITARIO }\end{array}$ & TELEVISIÓN & RADIO & COMENTARIO \\
\hline Daneses & No hay & No hay & $\begin{array}{l}\text { Se accede sin dificultad a la } \\
\text { radio y TV danesa. Existen } \\
\text { proyectos de crear programa- } \\
\text { ción propia. }\end{array}$ \\
\hline Serbios & $\begin{array}{l}1 \text { hora diaria } \\
\text { (Lunes a Vier- } \\
\text { nes) y } 3 \text { fines de } \\
\text { semana en Sorbi- } \\
\text { ches Studio } \\
\text { Baulzen }\end{array}$ & $\begin{array}{l}1 \text { hora los lunes } \\
\text { dedicada a jóve- } \\
\text { nes en la Radiop } \\
\text { Satkurba (se re- } \\
\text { pite el martes } \\
\text { por la Ostdents- } \\
\text { her Rundfunk } \\
\text { Brandenburg) }\end{array}$ & $\begin{array}{l}\text { Sólo programación cultural, in- } \\
\text { formación y educación en len- } \\
\text { gua serbia en Saxony y Bran- } \\
\text { denburgo. Además, se añade } \\
\text { programación emitida por te- } \\
\text { levisiones y radios locales, } \\
\text { como la ORB. En este caso } \\
\text { suele ser folklore, sobre todo. }\end{array}$ \\
\hline Frisones & $\begin{array}{l}\text { A través de la } \\
\text { Norddeutscher } \\
\mathrm{R} \text { u n d f u n k } \\
\text { (NDR), máximo } \\
4 \text { horas/año }\end{array}$ & $\begin{array}{l}\text { Máximo } 4 \text { ho- } \\
\text { ras/año }\end{array}$ & $\begin{array}{l}\text { Programación desde } 1989 \text { en } \\
\text { lengua frisona. Hay que aña- } \\
\text { dir lo emitido por emisoras de } \\
\text { radio y TV locales, como la } \\
\text { Telewindow. }\end{array}$ \\
\hline Sintis y gitanos & No hay & $\begin{array}{l}\text { Sólo en Berlín } \\
\text { un programa a } \\
\text { través de la Sen- } \\
\text { der Freies Ber- } \\
\text { lin (SFB) cono- } \\
\text { cida como" } \\
\text { Multikulti". }\end{array}$ & $\begin{array}{l}\text { Se justifica por la dispersión } \\
\text { geográfica, que haría muy cos- } \\
\text { tosa y casi imposible cualquier } \\
\text { tipo de programación. }\end{array}$ \\
\hline
\end{tabular}

15 Esta institución se creó en 1991 y tiene como labores asignadas más destacadas, la defensa y representación de los intereses de este colectivo, incluida la posibilidad de tener iniciativas políticas y legislativas. Además, tiene un papel activo en todas las acciones encaminadas a compensaciones económicas, conmemoraciones, etc., referidas a las actuaciones cometidas por el estado contra este grupo durante la etapa del gobierno nazi en Alemania. 
No obstante, y a pesar de que la programación en lenguas minoritarias y específica para estos grupos es muy escasa, como puede observarse, cabe decir que la tolerancia y el pluralismo cultural en Alemania son la tónica general, incluso por encima de otros países en los que la programación es mayor. Ese pluralismo cultural, en relación a las minorías nacionales reconocidas legalmente, no olvidemos que está garantizado, además de por la Constitución, por las diferentes Land Media Acts.

\section{Moldavia: un ejemplo paradigmático (referencia: acfc/sr (2000) 2)}

Moldavia es un país que por sus características especiales ${ }^{16}$ asumió sus compromisos con los grupos minoritarios nacionales desde la perspectiva de la necesidad de realizar una reforma profunda de sus estructuras estatales, sociales y políticas que cristalizase en un sistema jurídico acorde con los fundamentos democráticos y que terminara con los problemas nacionalistas en su interior.

Ya la propia Declaración de Independencia (agosto de 1991) establecía que el estado garantizaría "el ejercicio de los derechos sociales, económicos, culturales y de las libertades políticas de todos los ciudadanos de la República de Moldavia, incluyendo las personas pertenecientes a los grupos nacionales étnicos, lingüísticos y religiosos..." La Constitución, aprobada en julio de 1994, se hacía eco de la misma idea en iguales términos. Más adelante, en 1995, entró en vigor la Ley de Prensa, que prohíbe cualquier tipo de manifestación que incite al odio nacional y religioso (Ley 564 - XIX). También en la misma línea hay que citar la Ley del Audiovisual y una serie de decretos presidenciales relativos al desarrollo de la cultura nacional de rusos, ucranianos, búlgaros y judíos, principalmente. Lo que no existe es una ley específica dedicada a la protección de las minorías nacionales, aunque ha habido diversos intentos de aprobar una desde 1995. Por lo que se refiere al Convenio Marco para la Protección de las Minorías Nacionales, lo firmó en 1995 y lo ratificó un año después.

16 El 70\% de la población de este pequeño país es de origen rumano. Este grupo, al amparo de la perestroika, desde 1989, formó el llamado Frente Popular Nacionalista y reivindicó una serie de cuestiones culturales, entre las que destacaba la adopción del moldavo (rumano) como lengua oficial. A partir de aquí hubo una sucesión de problemas políticos y armados en los que los grupos minoritarios (rusos, gagazúes, turcos cristianos/ortodoxos y ucranianos), comenzaron a tener protagonismo desde su propia condición de minorías nacionales. El parlamento moldavo declaró la independencia en 1991 (como una primera fase para la posterior unión con Rumanía), lo que llevó a una guerra civil con los grupos rusos, que también proclamaron su independencia ya que no aceptaban la unión con Rumanía. En 1992 firmaron la paz, abandonando unos sus intenciones de unión con el país hermano y los otros su aspiraciones independentistas, aunque sí mantuvieron una amplia autonomía. Finalmente, el 6 de marzo de 1994 se realizó un referéndum que confirmó la independencia. Ya como país independiente consolidado, Moldavia ingresó en la ONU en 1992. Meses después se adhería también a la CEI con la misma condición de república independiente. Y en 1995 ingresó en el Consejo de Europa (el primer estado de la CEI en ser admitido). 
Las minorías reconocidas, según el Departamento Gubernamental de Análisis Estadísticos y Sociológicos, son - por orden de importancia - ucranianos, rusos, gagazúes ${ }^{17}$, búlgaros, bielorrusos, gitanos, alemanes y polacos. Teniendo en cuenta que los cuatro últimos grupos reseñados no llegan ni al 2\% de la población. También hay que señalar que se han creado una serie de asociaciones que representan a otros grupos minoritarios, pero que prácticamente no tienen presencia efectiva entre la población.

Desde los poderes públicos, se aprobó la creación de una serie de organismos que tienen un papel muy activo en la defensa y promoción de los derechos de las minorías nacionales, como por ejemplo el llamado Departamento de Relaciones Nacionales y funcionamiento de las Lenguas, la Dirección General para la Promoción de la Lengua Oficial ${ }^{18}$, o la Comisión Presidencial para las Relaciones Interétnicas.

Por lo que se refiere a los medios de comunicación, la presencia de los grupos minoritarios viene marcada, primero por las singularidades antes descritas. En segundo lugar, por la situación económica del país, que aunque pareció estabilizarse a partir de 1995, fue más una ilusión que una realidad. Tercero, por el problema secesionista de la zona del Transdniéster. Cuarto, por la dispersión de sus grupos minoritarios, con la excepción de la población gagazúe que se distribuye compactamente en la demarcación territorial llamada Gagazoues Yeri, con una presencia de este grupo superior al $70 \%$ del total. Y, en quinto lugar, se constatan las dificultades de recepción de las señales de televisión en varias zonas del país, lo que dificulta en gran medida la utilización de este medio. Todo lo anterior, lleva a que no exista una política concreta de programación específica para la televisión. El gobierno está más empeñado en conseguir, en primer lugar, la recepción general en el país de la Reseau TV (televisión de la República de Moldavia) y de otras grandes cadenas, como la SUN TV, con 26 canales o la NIT, más reducida.

En cuanto a la radiodifusión, sí existen programas en lenguas minoritarias, aunque sólo en ruso, ucraniano, búlgaro, gagazúe, yiddish y romaní.

\section{La federación rusa (acfc/sr (99) 15}

La Ferderación de Rusia es quizás uno de los ejemplos que cualquier laboratorio desearía en lo que a existencia de grupos nacionales minoritarios se

17 Los gagazúes son un pueblo de origen turco y religión cristiana que habita en el sur del país desde mediados del siglo XIX.

18 No se puede olvidar que la lengua oficial, aunque es el moldavo, en identidad lingüística con el rumano, es estrictamente una lengua propia de una minoría, que podríamos decir mayoritaria.. Es ésta una singularidad especial aunque no única en los nuevos países creados a partir de la esfera de la antigua Unión Soviética. Por ejemplo, en el mismo caso está Estonia, donde la mayoría de la población habla ruso y no estonio, la lengua oficial. 
refiere. Sobre una población de 140 millones de habitantes, se encuentran diseminados más de 170 pueblos distintos que en total constituyen en torno al $20 \%$ del total de la población. Ahora bien, el gobierno de este estado autodenominado poliétnico considera que de todos estos diferentes pueblos, sólo 23 de ellos formarían parte de lo que denominan una minoría nacional clásica. Aquí quedan incluidos, entre otro, los azerbayanos, armenios, lituanos, moldavos, polacos, ucranianos, tajikssistanos, kazajistanos, etc.

Si hacemos un breve repaso a la situación legal de las minorías nacionales el panorama que observamos podría considerarse satisfactorio. Así, la Constitución de la Federación rusa reconoce igualdad de derechos y libertades para todos los ciudadanos de la Federación, lo que, en teoría, permite a dichos ciudadanos la utilización de sus lenguas propias, la posibilidad de recibir educación acorde con sus orígenes... Para propiciar el desarrollo efectivo de la Carta Magna se han aprobado, asimismo, una serie de leyes, como la Ley de Autonomía Nacional y Cultural, la Ley de Garantías de los Derechos de los pequeños pueblos indígenas o la Ley sobre Medios de comunicación, entre otras. Junto a todo ello, la Federación rusa también ha ratificado una serie considerable de tratados internacionales, incluido el Convenio Marco para la Protección de las Minorías Nacionales.

Ahora bien, no obstante todo ese cuerpo legal, la realidad no es tan positiva como ese conjunto de leyes y tratados nos pueden hacer creer. Para empezar, en la legislación vigente no existe una definición de lo que la Federación considera minoría nacional. La consecuencia inmediata de ello es que las autoridades no poseen una lista de estos grupos. Ante este vacío, se hace fácil entender la dificultad que entraña aplicar las leyes y convenios ratificados.

Por lo que se refiere a los medios de comunicación, un país con una población y un espacio tan amplios, lógicamente, presenta una variedad importante de publicaciones y medios audiovisuales. La Ley de Medios de Comunicación, en consonancia con la Constitución que reconoce la libertad de pensamiento y palabra (art. 29), no contempla ningún tipo de limitación, excepto la prohibición de promover la intolerancia religiosa y nacional, así como el odio. Existen en torno a 400 periódicos y revistas que se publican en 59 diferentes lenguas, repartidos entre las distintas regiones que forman la Federación. Pero, a pesar de la existencia de éstos, la realidad viene dominada por las publicaciones que podríamos denominar rusas, siendo el resto, en muchos casos, más una anécdota que una realidad. Esto se agudiza, lógicamente, en el caso de los grupos minoritarios más pequeños. O por ejemplo, con los gitanos, que prácticamente carecen de presencia comunicacional en la Federación rusa.

En cuanto a la radio, hay programación en 43 lenguas; mientras que la televisión emite programas en 33 distintos idiomas. Pero nos referimos, tanto en el caso de la radio como la televisión, a programación regional o local, muy restringida y con un alcance limitado. La televisión que podríamos llamar rusa, es 
decir, la que emite para todo el territorio nacional, sólo lo hace en ruso y la presencia de las minorías nacionales se limita, en la mayoría de los casos, a ser más negativa que positiva. A ello hay que añadir que tradicionalmente en la antigua Unión Soviética y, subsiguientemente hoy en la Federación de Rusia, incluso el término minoría nacional tiene unas connotaciones negativas para la mayoría de la población. Y esta imagen, lejos de ser combatida por los medios de comunicación, en bastantes casos es consentida, cuando no potenciada.

Finalmente, podemos añadir que frente a esas cifras citadas en el informe presentado por la Federación rusa ante el Comité de Ministros del Consejo de Europa, que sugieren una cara amable, tenemos también otras varias realidades que no pueden ser obviadas. En primer lugar, todo lo referente al tema de las minorías es competencia exclusiva de la Federación. Ello quiere decir que en los escalones inferiores, como pueden ser los regionales o locales, existe un vacío importante y que la existencia de publicaciones o programación radiotelevisiva en la lengua de dichas regiones, por ejemplo, no puede confundirse con presencia "nacional" de los grupos minoritarios. Además, las emisiones que reciben en esas lenguas suelen provenir del otro lado de la frontera, en el caso de los grupos minoritarios situados en los bordes del territorio. Es decir, en puridad no estamos hablando de emisiones específicas para las minorías, sino de programación de otro país, cuya señal puede captarse en el territorio de la federación en el cual vive un grupo minoritario perteneciente al ámbito cultural y lingüístico del país emisor. Y más importante aún. No podemos olvidar que existen importantes regiones de este vasto estado en las que los derechos de las minorías nacionales se han visto claramente vulnerados. No es sólo el llamativo y quizás más conocido caso de Chechenia, sino en general es toda la zona del Cáucaso y Asia Central la que tiene problemas en este sentido. Y por lo que se refiere a los medios de comunicación, lejos de tener presencia en ellos, la cultura y la lengua de los pequeños pueblos indígenas de esta zona corre el peligro de perderse debido a un proceso de asimilación a lo ruso o rusificación.

Por todo lo anterior, podemos concluir que la Federación de Rusia constituye un ejemplo, no precisamente positivo, dentro del tema que tratamos.

\section{PAÍSES SIN MINORIAS RECONOCIDAS: MALTA, SAN MARINO Y LIECHTENSTEIN (Referencias: ACFC/SR (99) 12; ACFC/SR (99) 1; ACFC/SR (99) 4)}

Existe un bloque de países, como Malta, Liechtenstein y San Marino que, si bien han ratificado el Convenio Marco para la Protección de las Minorías Nacionales, no reconocen la existencia de grupos minoritarios nacionales numéricamente significativos en su territorio. Por ello, no puede existir, obviamente ningún tipo de programación específica para estos grupos. 
Estos países, a pesar de no tener minorías nacionales, han decidido ratificar la Convención porque lo consideran un acto de solidaridad, justificado por los propios contenidos del Convenio Marco.

\section{A modo de epilogo}

No parece que quepan dudas de que la diversidad cultural es una muy vieja y arraigada idea en Europa, debido entre otras razones a que Europa, desde tiempos muy remotos, ha sido un continente caracterizado por la migración. Esta característica típicamente europea se ha visto enriquecida en el siglo XX por una nueva emigración, diferente a la anterior, que nos ha convertido en receptores de grandes masas de ciudadanos procedentes del llamado Tercer Mundo. Esa realidad ha convertido Europa en un continente, tanto multicultural como multiétnico, caracterizado por la existencia de la diversidad cultural y obligado a conciliar ésta entre todos los ciudadanos y en los diferentes escalones de la política.

A lo largo de este estudio se ha puesto de manifiesto que las sociedades contemporáneas democráticas no se cuestionan el incluir entre sus presupuestos ideológicos teóricos y entre sus postulados constitucionales prácticos la protección especial de sus minorías nacionales. Pero no es sólo la inclusión de esta realidad lo importante. Lo fundamental es que el propio concepto de protección de dichas minorías nacionales ha experimentado un cambio muy positivo y en algunos aspectos, incluso, espectacular en los últimos veinte años. De un amparo general, basado en el conjunto de la sociedad, hemos pasado a una defensa activa que incluye la promoción y el desarrollo de estos grupos nacionales determinados. Las nociones de identidad, cultura específica, religión, derecho a la participación en la vida pública y acceso a los medios de comunicación, son ahora presupuestos centrales en la nueva comprensión de los derechos de las minorías nacionales. Es decir, frente al modelo clásico de integracionismo Europa occidental se mueve bajo lo que se conoce como el modelo de participación o "concepto de Lijphart", que defiende la coexistencia pacífica de los distintos grupos bajo provisiones constitucionales que aseguran el reconocimiento de su realidad diferenciada y les confieren el derecho de representación propia y de participación en la vida pública $\mathrm{y}$ en los órganos de gobierno que les competa (SISK, 1996).

\section{Bibliografia utilizada}

- COSTA-LASCOUX, J., 1995: "Multiculturalism and public policy" en Difference and cultures in Europe.

- KYMLICKA, W., 1996: Ciudadanía multicultural, Barcelona, Paidós.

- LAMO DE ESPINOSA, E., 1995: "Fronteras culturales" en Culturas, estados, ciudadanos. Una aproximación al multiculturalismo en Europa, Madrid, Fund. Ortega y Gasset. 
- PHILlIPS, A.: The Framework Convention for the Protection of National Minorities: A Policy Analysis, 2002, Londres, Minority Rights Group International, MRG.

- POMIAN, K., 1990: L'Europe et ses nations, París, Gallimard.

- REQUEJO, P., 2000: Democracia parlamentaria y principio minoritario. La protección constitucional de las minorías parlamentarias, Barcelona, Ariel.

- SARTORI, G., 2001: La sociedad multiétnica. Pluralismo, multiculturalismo y extranjeros, Taurus, Madrid.

- SISK, T.D., 1996: Power Sharing and International Mediation in Ethnic Conflicts, Washington, US Institute of Peace.

- WALZER, M., 1997: Las esferas de la justicia. Una defensa del pluralismo y la igualdad, México, Fondo de Cultura Económica.

- YACOUB, J., 1998: Les minorités dans le monde, París, Desclée de Brouwer.

\section{$\underline{\text { Informes del consejo de europa utilizados }}$}

- Report submitted by Austria; Ref.: ACFC/SR (2000) 3

- Report submitted by Germany; Ref.: ACFC/SR (99) 17

- Report submitted by Hungary; Ref.: ACFC/SR (99) 10

- Report submitted by Italy; (Ref.: ACFC/SR (99) 7

- Report submitted by Liechtenstein; Ref.: ACFC/SR (99) 4

- Report submitted by Malta; Ref.: ACFC/SR (99) 12

- Report submitted by Moldova; Ref.: ACFC/SR (2000) 2

- Report submitted by Russian Federation; Ref.: (ACFC/SR (99) 15

\section{Otros informes utilizados}

- Annual report. Human Right Developments in 1998, 1999, Viena, International Helsinki Federation for Human Right (IHF).

- Framework Convention for the Protection of National Minorities. Collected texts, 2nd edition, Estrasburgo, Consejo de Europa, 2001.

- Report by Martii Ahtisari, Jochen Frowein y Marcelino Oreja (Austria), aprobado en París el 8 Septiembre de 2000.

- Second Report on Austria, 2000, European Commission againts Racism and Intolerance (Ref.: CRI (2001) 3).

- World Directory of Minorities, 1997, Londres, Minority Rights Group International. 